\title{
Spin polarized electric currents in semiconductor heterostructures induced by microwave radiation
}

\author{
C. Drexler, ${ }^{1}$ V.V. Bel'kov, ${ }^{2}$ B. Ashkinadze,${ }^{3}$ P. Olbrich,${ }^{1}$ C. Zoth,${ }^{1}$ V. Lechner, ${ }^{1}$ Ya.V. Terent'ev, ${ }^{2}$ \\ D.R. Yakovlev, ${ }^{2,4}$ G. Karczewski ${ }^{5}$, T. Wojtowicz, ${ }^{5}$ D. Schuh, ${ }^{1}$ W. Wegscheider, ${ }^{1}$ and S.D. Ganichev ${ }^{1}$ \\ 1 Terahertz Center, University of Regensburg, 93040 Regensburg, Germany \\ ${ }^{2}$ A.F. Ioffe Physical-Technical Institute, Russian Academy of Sciences, 194021 St. Petersburg, Russia \\ ${ }^{3}$ Solid State Institute, Technion-Israel Institute of Technology, 32000 Haifa, Israel \\ ${ }^{4}$ Experimental Physics 2, TU Dortmund University, 44221 Dortmund, Germany, and \\ ${ }^{5}$ Institute of Physics, Polish Academy of Sciences, 02668 Warsaw, Poland
}

\begin{abstract}
We report on microwave ( $\mathrm{mw}$ ) radiation induced electric currents in (Cd,Mn) $\mathrm{Te} /(\mathrm{Cd}, \mathrm{Mg}) \mathrm{Te}$ and InAs/(In,Ga)As quantum wells subjected to an external in-plane magnetic field. The current generation is attributed to the spin-dependent energy relaxation of electrons heated by mw radiation. The relaxation produces equal and oppositely directed electron flows in the spin-up and spin-down subbands yielding a pure spin current. The Zeeman splitting of the subbands in the magnetic field leads to the conversion of the spin flow into a spin-polarized electric current.
\end{abstract}

PACS numbers: 73.21.Fg, 72.25.Fe, 78.67.De, 73.63.Hs

The discovery of microwave induced oscillations in the resistivity of a two-dimensional electron gas (2DEG) attracted growing attention to an electron magnetotransport in semiconductor nanostructures subjected to mw radiation, see, e.g., [1, 2]. The experimental observation of mw-induced effects stimulated much theoretical interest (see [3] and references therein) since they provide information which is complementary to conventional transport. In addition, the mw-induced effects offer new ways for developing sensitive microwave detectors [4]. All these effects have been observed applying an external magnetic field perpendicularly to a 2DEG plane.

In this Letter we demonstrate that microwave radiation induces a novel electron transport effect also under an in-plane magnetic field, i.e., in the geometry that excludes the cyclotron motion and Landau quantization of the two-dimensional electrons. The effect is caused by an asymmetric spin-dependent electron energy relaxation of the 2DEG heated by mw radiation [5, 6]. In an external magnetic field, this process results in a spin-polarized electric current. The mw-induced currents are observed in two different semiconductor systems: diluted magnetic semiconductor (DMS) $(\mathrm{Cd}, \mathrm{Mn}) \mathrm{Te} /(\mathrm{Cd}, \mathrm{Mg}) \mathrm{Te}$ quantum wells (QWs) and InAs/(In,Ga)As QWs. In these structures the spin-polarized electric currents are enhanced due to either the presence of magnetic $\mathrm{Mn}^{2+}$ ions [6] or the strong spin-orbit coupling in InAs [5].

Two $n$-type doped QW structures have been grown by molecular-beam epitaxy on (001)-oriented substrates. The first sample has a digital alloy DMS QW [7], which is a $10 \mathrm{~nm}$-wide $\mathrm{CdTe} \mathrm{QW}$ containing three monolayers of $\mathrm{Cd}_{0.86} \mathrm{Mn}_{0.14} \mathrm{Te}$ (see inset in Fig.(1). The effective average concentration of $\mathrm{Mn}$ is $\bar{x}=0.013$. The sample is $\delta$-doped with Iodine donors introduced into the top $\mathrm{Cd}_{0.76} \mathrm{Mg}_{0.24} \mathrm{Te}$ barrier at a $15 \mathrm{~nm}$ distance from the QW. The 2DEG has the density $n=6.2 \times 10^{11} \mathrm{~cm}^{-2}$ and the mobility $\mu=1.6 \times 10^{4} \mathrm{~cm}^{2} /$ Vs at a temperature

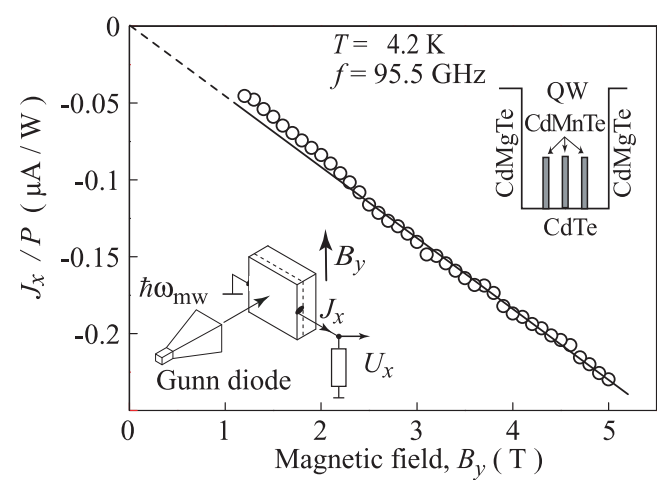

FIG. 1: Magnetic field dependence of the mw-induced signal $J_{x}$ for DMS (Cd,Mn)Te/(Cd,Mg)Te QW sample (circles). Insets show the experimental geometry and the structure sketch.

$T=4.2 \mathrm{~K}$. For further properties see Ref. [6]. The second sample is a non-magnetic InAs QW consisting of a $20 \mathrm{~nm}$ wide $\mathrm{In}_{0.75} \mathrm{Ga}_{0.25} \mathrm{As} / \mathrm{In}_{0.75} \mathrm{Al}_{0.25}$ As structure with a $4 \mathrm{~nm}$ InAs channel embedded in InGaAs. It is Silicon $\delta$-doped with $n=1 \times 10^{12} \mathrm{~cm}^{-2}$ and $\mu=7 \times 10^{4} \mathrm{~cm}^{2} / \mathrm{Vs}$ in the $2 \mathrm{DEG}$ at $T=4.2 \mathrm{~K}$.

In the mw-induced current experiments, samples of $5 \times 5 \mathrm{~mm}^{2}$ size with a pair of ohmic contacts along the direction $x \|[1 \overline{1} 0]$ are used. The experimental geometry is sketched in the inset of Fig.1 The samples were placed into an optical cryostat and a split-coil superconducting magnet yielding a field $B_{y} \|[110]$ with a strength up to $5 \mathrm{~T}$. The sample temperature was varied from $1.8 \mathrm{~K}$ to $150 \mathrm{~K}$. The microwave radiation was generated by two Gunn oscillators operating at frequencies, $f$, of $95.5 \mathrm{GHz}$ or $60 \mathrm{GHz}$. The radiation is guided through the cryostat windows on the sample at normal incidence utilizing a horn antenna and two parabolic mirrors. The distance between the horn antenna output and the sample was about $320 \mathrm{~mm}$. The incident $\mathrm{mw}$ power of about $0.1 \mathrm{~mW}$ 


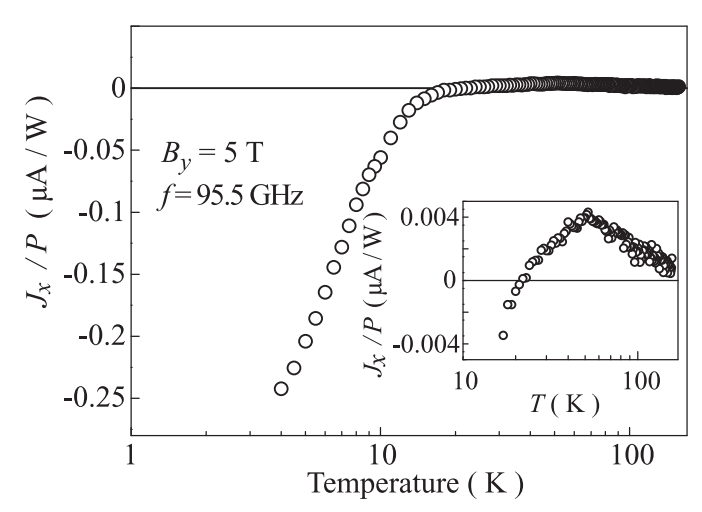

FIG. 2: Temperature dependence of the mw-induced photocurrent $J_{x}$ in the DMS $(\mathrm{Cd}, \mathrm{Mn}) \mathrm{Te} /(\mathrm{Cd}, \mathrm{Mg}) \mathrm{Te} \mathrm{QW}$. Inset shows the zoom of the zero crossing region.

was modulated by a p-i-n switch at $970 \mathrm{~Hz}$. The mwinduced current in the unbiased structure, $J_{x}$ (perpendicular to $B_{y}$ ) was measured with lock-in technique via the voltage drop $U_{x}$ across a $1 \mathrm{M} \Omega$ or $50 \Omega$ load resistors.

Figure 1 shows the current $J_{x}$ induced by $95.5 \mathrm{GHz}$ irradiation of the DMS QW at $T=4.2 \mathrm{~K}$ as a function of the magnetic field strength. The current increases with $B_{y}$ and reverses its sign as the direction of $B_{y}$ changes (not shown). As the lattice temperature increases from $2 \mathrm{~K}$ to $50 \mathrm{~K}$, the current value decreases by more than two orders of magnitude and the signal reverses its sign at $T \approx 20 \mathrm{~K}$, see Fig.2 Similar results have also been obtained for the mw-frequency of $60 \mathrm{GHz}$.

Figure 3 demonstrates $J_{x}\left(B_{y}\right)$ dependences for the non-magnetic InAs/(In, Ga)As QW obtained at $95.5 \mathrm{GHz}$ irradiation for several $T$. The mw-induced current is detected in the temperature range from $1.8 \mathrm{~K}$ to $65 \mathrm{~K}$. The current depends linearly on $B_{y}$, however, contrary to the DMS sample, it decreases smoothly with rising $T$ and does not change the sign (see inset in Fig.3). It will be shown below that such a significant difference in the $J_{x}(T)$ dependences is caused by various temperature behavior of the Zeeman spin splitting in both materials, which for DMS structures is strongly influenced by the polarization of Mn ions [ $[, 9]$.

Our findings can be well understood in the frame of the recently proposed model for the spin-dependent asymmetric energy relaxation of a nonequilibrium (mwheated) electron gas [5]. Free electrons absorbing microwave radiation, are excited to higher energy states, then they relax into an equilibrium state by emitting phonons. Figure 4 sketches the energy relaxation processes of hot electrons in two spin subbands $\left(s_{y}= \pm 1 / 2\right)$ which are split in an external magnetic field due to the Zeeman effect. The energy relaxation is shown by bend arrows. Usually, the energy relaxation via scattering by phonons is considered to be spin-independent. However, in gyrotropic media, like CdTe- and InAs-based QWs, the spin-orbit interaction adds an asymmetric spin-

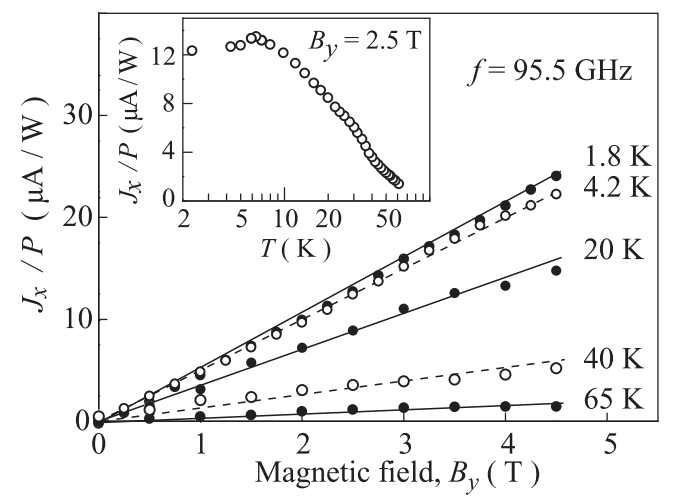

FIG. 3: Magnetic field dependence of the mw-induced electric current $J_{x}$ in the InAs/(In,Ga)As QW. Its temperature dependence is shown in the inset.

dependent term to the scattering probability with the matrix element being proportional to $\left[\boldsymbol{\sigma} \times\left(\boldsymbol{k}+\boldsymbol{k}^{\prime}\right)\right]$ (note, that we assume presence of the structure inversion asymmetry only) [5]. Here, $\sigma$ is the axial vector composed of the Pauli matrices; $\boldsymbol{k}$ and $\boldsymbol{k}^{\prime}$ are the initial and final electron wave vectors. Thus, the electron transitions to positive and negative $k_{x}^{\prime}$-states occur with different probabilities leading to an imbalance in the distribution of carriers between positive and negative $k_{x}$-states in both subbands $\left(s_{y}= \pm 1 / 2\right)$. This is shown by bent arrows of different thicknesses for both subbands in Fig. 4. In the absence of an external magnetic field the asymmetry of the electron-phonon interaction results in a spin current, but not in an electric current since the oppositely directed electron fluxes, $i_{ \pm 1 / 2}$, in spin subbands $s_{y}= \pm 1 / 2$ are of equal strength and, therefore, compensate each other. The external magnetic field leads to the Zeeman splitting of the $s_{y}$ subbands. As a result, the electron densities in the spin-up and spin-down subbands become different, and the fluxes $i_{ \pm 1 / 2}$ do not longer compensate each other yielding a net electric current. Obviously, the current is spin polarized and its value is proportional to the Zeeman splitting energy, $E_{Z}$. Such a physical mechanism of the mw-induced current generation explains the significantly different behavior of the currents in the DMS and non-magnetic structures.

In DMS structures the exchange interaction of electrons with magnetic ions results in the giant Zeeman spin splitting of the conduction band, $E_{Z}$, and amplifies spindependent phenomena, see, e.g., [8-10]. Moreover, $E_{Z}$ has a remarkable temperature dependence [10]

$$
E_{\mathrm{Z}}=g \mu_{\mathrm{B}} B+\bar{x} S_{0} N_{0} \alpha \mathrm{B}_{5 / 2}\left(\frac{5 \mu_{\mathrm{B}} g_{M n} B}{2 k_{\mathrm{B}}\left(T_{M n}+T_{0}\right)}\right),
$$

where $k_{\mathrm{B}}$ is the Boltzmann constant and $\mu_{\mathrm{B}}$ the Bohr magneton. The first term is the intrinsic spin splitting with the electron $g$-factor $g=-1.64$ for CdTe QWs [1]]. The second one describes the electron exchange interac- 


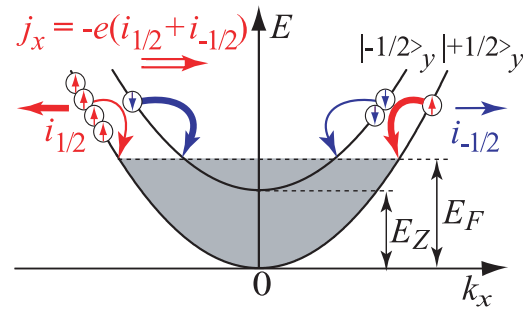

FIG. 4: Model of the mw-induced generation of spin-polarized current. See details in the text.

tion with $\mathrm{Mn}^{2+}$ ions, where $g_{M n}=2$ is the Mn $g$-factor and $T_{M n}$ the Mn-spin system temperature. The parameters $S_{0}$ and $T_{0}$ account for the Mn-Mn antiferromagnetic interaction, $\mathrm{B}_{5 / 2}$ is the modified Brillouin function and $N_{0} \alpha=220 \mathrm{meV}$ is the exchange integral [9]. The sign inversion of $J_{x}(T) \propto E_{Z}$, shown in the inset of Fig.2, directly follows from Eq. (1). Indeed, $E_{Z}$, reverses its sign upon temperature variation due to opposite signs of $g$ and $N_{0} \alpha$. This explains the experimental data shown in Fig.2. It is worthwhile to note that in our previous photoluminescence study, the sign inversion of the Zeeman splitting was detected at $T \approx 40 \mathrm{~K}[6]$. This temperature is higher than the inversion temperature detected in the mw-induced current $(T=20 \mathrm{~K})$. We attribute this difference to the mw-heating of the Mn spin system above $T$, as reported in [6, 12].

In contrast to our DMS QWs, the sign of the Zeeman splitting in the non-magnetic InAs-based structure $\left(E_{\mathrm{Z}}=\right.$ $g \mu_{\mathrm{B}} B$ ) does not change (for bulk InAs $g \approx-15[13]$ ). Hence, the temperature dependence of the mw-induced current shown in the inset of Fig. [3 is mostly determined by the spin up/down subband population (Boltzmann factor) [5]. We notice, that a similar $J_{x}(T)$ behavior was also observed at terahertz $(\mathrm{THz})$ frequencies [5, 6]. The mechanism of the current generation for $\mathrm{THz}$ - and $\mathrm{mw}-$ excitation is the same, however, in the mw-range the electron gas heating for a fixed power is higher due to the larger Drude-absorption for smaller photon energies.

The signals detected in our experiments, in particular at low temperatures, are rather large (being of the order of $\mu \mathrm{V}$ ) and therefore, may contribute substantially to the magneto-transport phenomena studied under inplane magnetic fields [14]. For (001)-grown zinc-blende QWs, the spin-polarized current generation can only occur under application of an in-plane magnetic field: The photocurrent generation with $\boldsymbol{B}$ normal to the 2DEG plane geometry $(\boldsymbol{B} \| z)$ is forbidden by symmetry reasons. However, in [110]- and [113]-grown QWs, the effect becomes allowed for the $\boldsymbol{B} \| z$-geometry [15] and, therefore, may be of importance for the analysis of mw-induced current-voltage effects studied in magnetic fields applied perpendicularly to 2 DEG plane. To summarize, our work demonstrates a convenient method of spin polarized currents generation by microwave radiation of conventional
Gunn diodes. The direction and magnitude of such spinpolarized currents can be controllably varied by a proper combination of the crystallographic orientation and magnetic field direction as follows from the discussed physical mechanism for the current generation.

The financial support from the DFG (SFB 689 and SPP 1285), the Linkage Grant of IB of BMBF at DLR, Russian Ministry of Education and Sciences, and RFBR, is gratefully acknowledged. The research was partially supported by the EU within European Regional Development Found, through grant Innovate Economy (POIG.01.01.02-00-008/08) and by the Foundation for Polish Science through subsidy 12/2007.

[1] A. T. Hatke, M. A. Zudov, L. N. Pfeiffer, and K. W. West, Phys. Rev. Lett. 102, 066804 (2009).

[2] I. V. Kukushkin, M. Yu. Akimov, J.H. Smet, S. A. Mikhailov, K. von Klitzing, I. L. Aleiner, and V. I. Falko, Phys. Rev. Lett. 92, 236803 (2004).

[3] I. A. Dmitriev, M. Khodas, A. D. Mirlin, D. G. Polyakov, and M. G. Vavilov, Phys. Rev. B 80, 165327 (2009).

[4] P. S. Dorozhkin, S. V. Tovstonog, S. A. Mikhailov, I. V. Kukushkin, J. H. Smet, and K. von Klitzing, Appl. Phys. Lett. 87, 092107 (2005).

[5] S. D. Ganichev, V. V. Bel'kov, S. A. Tarasenko, S. N. Danilov, S. Giglberger, Ch. Hoffmann, E. L. Ivchenko, D. Weiss, W. Wegscheider, Ch. Gerl, D. Schuh, J. Stahl, J. De Boeck, G. Borghs, and W. Prettl, Nature Phys. 2, 609 (2006).

[6] S. D. Ganichev, S. A. Tarasenko, V. V. Bel'kov, P. Olbrich, W. Eder, D. R. Yakovlev, V. Kolkovsky, W. Zaleszczyk, G. Karczewski, T. Wojtowicz, and D. Weiss Phys. Rev. Lett. 102, 156602 (2009).

[7] J. Jaroszynski, T. Andrearczyk, G. Karczewski, J. Wróbel, T. Wojtowicz, E. Papis, E. Kaminska, A. Piotrowska, D. Popovic, and T. Dietl, Phys. Rev. Lett. 89, 266802 (2002).

[8] Spin Physics in Semiconductors, Ed. M. I. Dyakonov (Springer, Berlin, 2008).

[9] T. Dietl, in Handbook on Semiconductors, Vol. 3b, Ed. T. S. Moss (North-Holland, Amsterdam, 1994).

[10] J. K. Furdyna, J. Appl. Phys. 64, R29 (1988).

[11] A. A. Sirenko, T. Ruf, M. Cardona, D. R. Yakovlev, W. Ossau, A. Waag, and G. Landwehr, Phys. Rev. B 56, 2114 (1997).

[12] B. König, I. A. Merkulov, D. R. Yakovlev, W. Ossau, S. M. Ryabchenko, M. Kutrowski, T. Wojtowicz, G. Karczewski, and J. Kossut, Phys. Rev. B 61, 16870 (2000).

[13] O. Madelung, M.Schulz, and H. Weiss, Numerical Data and Factional Relationships in Science and Technology, Landolt-Börnstein, New Series, Group III, Part 17 a (Springer, Heidelberg, 1982).

[14] C. L. Yang, R. R. Du, L. N. Pfeiffer, and K. W. West, Phys. Rev. B 74, 045315 (2006).

[15] V. V. Bel'kov, P. Olbrich, S. A. Tarasenko, D. Schuh, W. Wegscheider, T. Korn, C. Schüller, D. Weiss, W. Prettl, and S. D. Ganichev, Phys. Rev. Lett. 100, 176806 (2008). 